\title{
Posicionamiento del investigador de enfermería en la utilización de la teoría fundamentada constructivista
}

\author{
Positioning of the nursing researcher in the \\ utilization of the constructivist grounded theory
}

\section{Posicionamento do pesquisador de enfermagem na utilização da teoria fundamentada construtivista}

\author{
H. Juanillo-Maluenda \\ ORCID: 0000-0002-0292-4333 \\ Departamento de Salud Pública, División de Disciplinas para el Desarrollo y Prevención de la Salud, Centro \\ Universitario de Ciencias de la Salud, Universidad de Guadalajara, Jalisco, México
}

Recibido: 13 enero 2018

Aceptado: 15 noviembre 2018

\section{Resumen}

Introducción: La teoría fundamentada ha tenido un fuerte auge en las ciencias de la salud, especialmente en la enfermería, producto de las ventajas que presenta para ser aplicada en problemas o situaciones de la disciplina y analizar diversos tópicos de interés en salud. Una de las vertientes más recientes, la teoría fundamentada constructivista, mantiene en la actualidad un amplio desarrollo por las características inherentes a este enfoque. Una de ellas, que la diferencia de las perspectivas predecesoras, es la posición del investigador en temáticas sociales aplicadas a grupos vulnerables como las personas mayores.

Desarrollo: El presente trabajo reflexiona en torno a los usos de la teoría fundamentada constructivista, la posición que debe adquirir el investigador que utilice este enfoque en investigaciones en enfermería, y las herramientas metodológicas con las que puede contar para cumplir este propósito al estudiar grupos vulnerables, como las personas mayores en un contexto latinoamericano.

Reflexiones finales. Uno de los aportes destacados de la TFC es posicionar al investigador en un rol clave en la co-construcción de la teoría, como partícipe del proceso de investigación en conjunto con sus participantes como sujeto de cambio social.

Palabras clave: Investigación cualitativa; teoría fundamentada constructivista; posicionamiento del investigador; personas mayores; México. 


\section{Abstract}

Introduction: Grounded theory has had an important boom within the health sciences, particularly in nursing, mainly as a result of the advantages it offers to address nursing problems or situations, as well as other diverse health topics of interest. One of its most recent variants, the constructivist approach, has maintained a steady development due to its distinctive characteristic of positioning the researchers towards the social issues related to the vulnerable groups, like the elders.

Development:The present work reflects upon the uses of the constructivist grounded theory, the positioning which the nursing researchers need to take, and the methodological tools which can be counted on in order to study vulnerable groups, such as the elders, within the Latin-American context.

Final Reflections: By positioning researchers and their subjects of study as active participants and theory co-builders in the constant process of social change, this methodology makes important contributions to the sciences of healthcare.

Keywords: Qualitative research; constructivist grounded theory; research positioning; elderly; Mexico.

\section{Resumo}

Introdução: A teoria fundamentada teve um forte auge nas ciências da saúde, especialmente na enfermagem, produto das vantagens que apresenta para ser aplicada em problemas ou situações da disciplina e analisar diversos tópicos de interesse em saúde. Uma das vertentes mais recentes, a teoria fundamentada construtivista, mantem na atualidade um amplo desenvolvimento pelas caraterísticas inerentes a este enfoque. Uma delas, que a diferencia das perspectivas predecessoras, é a posição do pesquisador em temáticas sociais aplicadas a grupos vulneráveis como as personas idosas.

Desenvolvimento: O presente trabalho reflete em volta dos usos da teoria fundamentada construtivista, a posição que deve adquirir o pesquisador que utilize este enfoque em pesquisas em enfermagem, e as ferramentas metodológicas com as que pode contar para cumprir este propósito ao estudar grupos vulneráveis, como as pessoas idosas em um contexto latino-americano.

Reflexiones finais. Uma das contribuições destacados da TFC é posicionar ao pesquisador em um papel chave na co-construção da teoria, como partícipe do processo de pesquisa em conjunto com seus participantes como sujeito de câmbio social.

Palavras chave: Pesquisa qualitativa; teoria fundamentada construtivista; posicionamento do pesquisador; pessoas idosas; México.

\section{Introducción}

La teoría fundamentada (TF) se posiciona a partir del paradigma interpretativista, el que se caracteriza por reconocer y narrar el significado de las experiencias humanas y sus acciones ${ }^{1}$. Este paradigma asume estar guiado por el conjunto de creencias y sentimientos del investigador sobre el mundo, respecto a cómo éste debe ser entendido y estudiado ${ }^{2}$. Asimismo, sus bases provienen del interaccionismo simbólico, específicamente desde las aportaciones sociológicas de la Escuela de Chicago, que fue bastante influyente en disciplinas como la psicología, enfermería y la sociología médica, por las ventajas para examinar interacciones sociales entre personas con enfermedades crónicas y la relación personal sanitario-paciente ${ }^{3}$.

La TF tiene su desarrollo a partir de sus fundadores; Glaser y Strauss ${ }^{4}$, quienes desarrollaron una metodología sistemática aplicada a entornos sociales a partir de enfoques inductivos, que les permitieron la generación de teoría a partir de los datos recolectados. Entre las pretensiones de esta perspectiva, se encontraban acortar las distancias entre las grandes tradiciones teóricas de las ciencias sociales y la investigación empírica ${ }^{5}$, principalmente, porque consideraban que las teorías utilizadas en la investigación eran a menudo inapropiadas e inadecuadas para los participantes bajo estudio ${ }^{6}$. En este punto es preciso realizar la primera aclaración respecto a este enfoque cualitativo, ya que la TF es una técnica analítica que se confunde muy a menudo con un enfoque teórico en sí mismo ${ }^{3}$. En esto también concuerda una de las 
exponentes más destacadas de la TF en los últimos 25 años, Kathy Charmaz, cuyos aportes han revitalizado este enfoque al ofrecer variadas posibilidades en las interpretaciones y análisis de contextos sociales ${ }^{2}$. Para Charmaz, la TF es un método para realizar investigación cualitativa que se enfoca en la creación de teorías mediante la construcción de análisis inductivo, a partir de los datos ${ }^{7}$. Este método se distingue por involucrar al investigador en el análisis de datos, mientras los recopila y las potencialidades que presenta al abordar grupos vulnerables, como las personas mayores.

La siguiente reflexión tiene por propósito describir las características de la TF constructivista (TFC) y argumentar la posición que deben tener los investigadores que utilicen este enfoque en investigaciones que aborden problemáticas relacionadas con las personas mayores como grupo vulnerable en sus contextos sociales.

\section{El devenir de la TF hacia el constructivismo}

Una manera de comprender la evolución que ha tenido esta perspectiva, es abordarla a partir de lo que

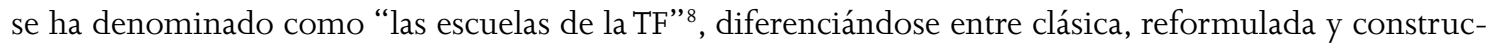
tivista. La primera está representada por los sociólogos Barney Glaser y Anselm Strauss, quienes a finales de la década de los sesenta articularon la metodología que plantearía las bases de la TF en el libro "El descubrimiento de la Teoría Fundamentada”, publicado en 1967, que significó una revitalización de la investigación cualitativa en sociología al presentar un modelo deductivo derivado de las grandes teorías macrosociológicas9. Esta propuesta se caracterizó por una fuerte inclinación positivista en el análisis, asumir las categorías como variables, construir una teoría a partir de cómo emergen los datos y reinterpretar los hallazgos. La segunda escuela la representa Strauss, pero ahora en compañía de la enfermera Juliet Corbin, quienes sustentan su enfoque en el libro "Bases de la investigación cualitativa. Técnicas y procedimientos para desarrollar teoría fundamentada”, publicado en 1990. En esta colaboración enfatizan en la necesidad de realizar interacciones entre los conceptos para interpretar los datos y brindar una explicación a partir de ellos, mediante el uso apropiado de métodos de investigación. Es bajo esta escuela que la TF se populariza en diversas disciplinas, principalmente en la enfermería. Ejemplo de esto es la aplicación de la TF en contextos sanitarios, al estudiar la vida cotidiana de las personas que viven y envejecen con discapacidades físicas o enfermedades crónicas de largo padecimiento ${ }^{10}$. Por último, la tercera escuela llamada constructivista, fue desarrollada por la socióloga Kathy Charmaz, a partir del debate en relación al rol que tienen los investigadores en sus estudios y que recibe una fuerte influencia del pensamiento constructivista ${ }^{11,12}$. En su libro "Construyendo teoría fundamentada. Una guía práctica para el análisis cualitativo”, publicado en el año 2006, establece un rediseño al modelo de la corriente clásica, al cuestionar sus bases objetivistas mediante un enfoque sistemático, que fomenta la integración de la experiencia subjetiva del investigador en la co-construcción de la teoría ${ }^{8}$. Los aportes de esta investigadora también se encuentran desde el área de las ciencias de la salud, al estudiar a profundidad a quienes padecen una enfermedad crónica por largo tiempo ${ }^{13}$, pero especialmente es reconocida por ser la primera investigadora, que describió y argumentó su trabajo explícitamente como constructivista ${ }^{14}$, que además ha tenido un fuerte auge entre investigadores sociales durante la última década ${ }^{8}$.

Como fue descrito, la TF ha presentado diversas reinterpretaciones en las corrientes posteriores a las de la escuela clásica. Pese a esto, Hood considera que es posible apreciar características comunes en los distintos enfoques, como las técnicas de recolección de datos, utilización del método de comparación constante, implementación del muestreo teórico, saturación teórica y el desarrollo inductivo de la teoría ${ }^{15}$.

\section{El enfoque de la TFC}

La perspectiva desarrollada por Charmaz, considera que existen múltiples realidades sociales que se vinculan a un mundo empírico; mantiene las estrategias inductivas de la TF desarrollada por sus predecesores, pero establece un alejamiento de la objetividad del investigador, por lo que reconfigura el rol del investigador en la construcción de los datos y la teoría ${ }^{16}$. Sus planteamientos para diferenciar la TFC, los 
argumenta a partir de las premisas objetivistas que primaron entre sus antecesores; a Glaser lo vincula al positivismo tradicional al suponer una realidad externa objetiva, observador neutral que descubre datos y reduccionista en su investigación; mientras que Strauss y Corbin asumen un objetivo externo al apuntar hacia una recopilación de datos, proponer un conjunto de procedimientos técnicos, y respaldar su verificación ${ }^{17}$.

Esta autora considera, desde una revisión más contemporánea, que la TFC mantiene las estrategias teóricas de la TF clásica y la TF reformulada, pero se diferencia, sobre todo por; asumir una epistemología relativista, adoptar una postura reflexiva de las relaciones y antecedentes con los participantes de la investigación, ubicar su investigación en condiciones históricas, sociales y situacionales de su producción y reconocer tanto al investigador como a sus participantes, los múltiples puntos de vista que presentan, sus roles y realidades diversas ${ }^{18}$. El rol que asume el investigador en la propuesta de Charmaz, es lo que implica realzar no sólo un trabajo interpretativo que debe tener el investigador, sino que también cómo éste juega un papel al construir una teoría sujeta a las motivaciones, experiencias pasadas, interacciones y perspectivas de los investigadores como actores sociales ${ }^{7}$.

Herramientas para el rol del investigador social en la TFC

La TFC presenta una notable diferencia con relación a las propuestas de sus antecesores en lo que respecta a cómo abordar los fenómenos sociales y asumir su rol como investigador social; ya que en la TFC existe un alejamiento de la tradicional postura objetiva del investigador, en la que adquieren un reconocimiento a su labor en la construcción tanto de los datos como de la teoría ${ }^{16}$. Para alcanzar estos propósitos, la TFC se nutre de la entrevista, de las notas de campo y de los memos teóricos.

El tipo de entrevista que se utiliza en la TFC es la entrevista intensiva ${ }^{7}$, que tiene como objetivo explorar en profundidad un tema, lo que implica un rol activo del entrevistador en la interpretación de las respuestas del entrevistado. Por su parte, las notas de campo tienen como utilidad describir personas, problemas, conversaciones y configuraciones ${ }^{19}$. Por último, los memos teóricos son una herramienta muy utilizada para realizar análisis cualitativo y se desarrollan desde la recolección de datos hasta la redacción del informe ${ }^{20}$. Para la TFC, los memos implican una conversación privada que los investigadores constructivistas tienen consigo mismos, cuando separan sus códigos y analizan lo que puede tener un significado; convirtiéndose así, en una herramienta fundamental en el desarrollo de análisis ${ }^{21}$. Para Böhm ${ }^{22}$, la redacción de memos requiere que los investigadores reflexionen en torno a los datos que emergen, con el objetivo de ir más allá de una elaboración meramente descriptiva. Con esto, la comparación constante de los memos se realiza con el fin de generar un insumo que pueda ser estudiado, refinado y que se utilice en cada paso que se realice en la investigación, con la meta final de elevar el nivel teórico del análisis emergente ${ }^{21}$. La utilidad de los memos desde esta perspectiva orienta al investigador a tomar conciencia de sus presuposiciones y cómo esto lidia con el proceso de investigación que lleva adelante; es por esto que el constructivismo fomenta la reflexión de los investigadores sobre sus propias interpretaciones y la de sus participantes ${ }^{7}$.

Para orientar al investigador en su rol de co-constructor de la teoría, la TFC demanda que se utilicen elementos integradores en la investigación, al establecer cuatro criterios de rigor; originalidad, credibilidad, utilidad y resonancia, que resguardan la calidad de los estudios y que se dirigen a través de preguntas que los investigadores deben ir planteándose a lo largo del proceso de investigación ${ }^{23}$.

De esta manera, la posición y experiencia que aporta el investigador social en torno a las temáticas de estudio, son parte de todo el proceso al ser miembros del campo de estudio al igual que los sujetos de investigación ${ }^{20,24}$. En términos conceptuales, esta posición se conoce como reflexividad, entendida como la participación de los investigadores en el examen continuo y explicación de cómo han influido ellos como parte del proceso de investigación ${ }^{25}$. De esta forma, la reflexividad resulta central para la TFC y que contrasta con el enfoque de la concepción original de la TF, ya que para los predecesores de la TFC, la construcción social provenía del mundo empírico, la cual enfatiza en la generalidad del proceso, no 
así de la reflexividad. Por esta razón desde la TFC, la reflexión que los investigadores de TF brindan a su método, otorga una amplia estrategia analítica de co-construcción de la realidad social ${ }^{26}$. De acuerdo con Hall y Callery ${ }^{27}$, esta interacción entre investigadores y participantes se hace más transparente, ya que considera la recopilación y análisis de los datos en torno a una construcción social del conocimiento, que fue descuidada en la TF clásica.

Para Charmaz ${ }^{7}$, quienes realicen investigaciones a partir de la TFC deben ser conscientes de sus presuposiciones y cómo pueden afectar a la investigación que dirigen, por ello, la reflexividad contribuye a fomentar las interpretaciones tanto de investigadores como de quienes participan en sus investigaciones, para alcanzar este nivel de conciencia.

También, esta conceptualización ha derivado a ser considerada una estrategia para alcanzar rigor en las investigaciones cualitativas, principalmente en el campo de la salud, ya que permiten una comprensión más amplia de la toma de decisiones metodológicas de los procesos ${ }^{28}$. Además, al escribir sobre la investigación y los procedimientos utilizados en ella, la reflexividad se convierte en un instrumento importante para transmitir lo que se hizo en el estudio, cómo se hizo y qué tan bien se hizo ${ }^{24}$; lo que, finalmente permite a los lectores juzgar la calidad del producto cualitativo generado ${ }^{27}$.

\section{Las personas mayores como población vulnerable}

En este punto, se plantearán problemas que hacen de la población adulta mayor un grupo social vulnerable, especialmente en lo que respecta a problemas transversales de salud en Latinoamérica.

Por grupos vulnerables se entienden aquellos procesos multidimensionales que confluyen en riesgo o probabilidad, de que los grupos sociales puedan ser lastimados ante cambios o mantenimiento de situaciones externas y/o internas; por ejemplo, esta vulnerabilidad podría expresarse como fragilidad e indefensión, como el desamparo desde el Estado que no contribuye a fortalecer la protección de quienes lo integran ${ }^{29}$.

En América Latina la población adulta mayor ha experimentado un aumento en la expectativa de vida, lo que implica que su población pase de un $11 \%$ a más de un $26 \%$, para el año $2050^{30}$. Gran parte de esta población arrastra brechas sociales en contextos de países empobrecidos en los años que vivieron su juventud y adultez, con escasos espacios a oportunidades de desarrollo. En los adultos mayores, estas inequidades son más marcadas porque se establecen a lo largo de la vida y dependen de complejos factores, que se interrelacionan entre sí, conforme al contexto en que los adultos mayores se desenvuelven ${ }^{31}$. Una de estas brechas sociales es la educación que, al configurar el nivel socioeconómico en el futuro, determina finalmente el estado de salud de las personas, las que además empeoran a medida que las poblaciones afectadas envejecen ${ }^{32}$. También, se debe mencionar que los problemas específicos que afectan a las personas mayores son distintos, de acuerdo con la región analizada, por lo que aquí se abarcarán problemas derivados de la falta de oportunidades que han tenido las personas mayores de gran parte de Latinoamérica y que las diferencian de los futuros adultos mayores de esta región, como ocurre en el caso de la educación. Precisamente, la educación se considera como uno de los principales determinantes de problemas sanitarios prioritarios, según reportes técnicos, por su responsabilidad en la aparición de demencias y el deterioro de la capacidad funcional en términos incapacitantes ${ }^{33}$.

En la región, las cifras en términos educacionales son desfavorables para las personas mayores, por ejemplo, el analfabetismo de este grupo en la mayoría de los países de la región oscila entre un 25\% y un $50 \%$; que presentan las cifras más altas en mujeres, población rural y países centroamericanos ${ }^{34}$. A su vez, el analfabetismo repercute en la vulnerabilidad socioeconómica futura de los adultos, que traspasa esta brecha por generaciones ${ }^{35}$. Por otra parte, el nivel socioeconómico de los adultos mayores es considerado un determinante social estructural sanitario, ya que configura las oportunidades de salud que tienen los grupos sociales ${ }^{36}$. Esto significa que las personas que pertenecen a niveles socioeconómicos vulnerables presentan peores condiciones de salud ${ }^{37}$, debido en gran parte a los costos elevados de la atención de la salud y la seguridad social que es variable en los países latinoamericanos ${ }^{34}$. 
La educación también se ha vinculado a inequidades en salud que se agudizan con los años. Uno de estos problemas sanitarios lo constituyen las demencias como la enfermedad de Alzheimer, que se asocia fuertemente a bajos niveles educacionales con un riesgo atribuible poblacional de $19 \%{ }^{32}$.

Otro ejemplo de inequidades en salud asociadas al nivel educacional en las personas mayores, lo representa la dependencia funcional, ya que presenta una asociación que afecta principalmente a mujeres, quienes manifiestan una expectativa de vida mayor que los hombres y tienen además, menores niveles de escolaridad ${ }^{38}$.

En vista que los adultos mayores, a partir de un problema transversal y latente aún en Latinoamérica, como el nivel educativo logrado, repercute en problemas cognitivos y funcionales, queda la inquietud respecto de cómo esperar que estos grupos vulnerables puedan colocar sus problemas en la agenda pública, con el objetivo de encontrar una solución y hacer valer sus derechos de acuerdo a las últimas convenciones que los protegen como grupo social vulnerable ${ }^{39}$.

Son estas las disyuntivas que se presentan en el resguardo de los derechos de las personas con deterioro de las capacidades funcionales o con demencias, ya que pese a estos nuevos marcos normativos, sus derechos siguen siendo ignorados como en el caso de las demencias ${ }^{40} \mathrm{o}$ en personas con capacidades funcionales disminuidas, en donde las instancias de participación democrática ante estas dificultades, no se explican, no se facilitan ni se aseguran sus canales de confluencia ${ }^{41}$.

Ante estas realidades, el investigador de enfermería que se involucra en estudiar problemas sociales que repercuten en la salud de la población, se torna un actor fundamental. Esto porque al inmiscuirse y formar parte del estudio de las problemáticas sociales, debe construir un cuerpo de conocimiento que explique y visibilice las exclusiones ante la comunidad científica, con el objetivo de difundir y repercutir o influir en la toma de decisiones políticas, para solucionar los problemas de las personas afectadas o vulneradas, como es el caso de las personas mayores.

El investigador de enfermería y su rol en el estudio de problemáticas relacionadas con las personas mayores La visibilidad que deben adquirir los asuntos implicados en el diario vivir de las personas mayores, como grupo social vulnerable y cómo sus temáticas se toman la agenda pública es un asunto pendiente. Principalmente, en las vías con que se cuentan en un Estado de derecho, para fomentar la participación de las personas mayores y definir por estos conductos, el cambio social que se requiere para mejorar las condiciones de vida de esta población. Esto se debe considerar no sólo para mitigar las problemáticas que los atañen, sino que además para brindar poder de decisión a poblaciones postergadas y excluidas, como es el caso histórico de las personas adultas mayores ${ }^{42}$.

Por los motivos descritos, es complejo encontrar canales reales para ejercer estos propósitos, dadas las barreras de los contextos sociales en que han vivido las personas mayores, como su baja escolaridad y las consecuencias que derivan de ellos, como las limitaciones en las capacidades funcionales y las demencias. Es en este punto que las subjetividades del investigador y su influencia en interpretar un fenómeno social, le permite desarrollar una mayor comprensión, aumentar la sensibilización a cuestiones éticas y morales, y emprender la emancipación personal y política del contexto que ha decidido estudiar ${ }^{2}$. Ante esta situación, el investigador no aborda un fenómeno conservando distancia de él, ya que no sólo debe tener muy claro qué está haciendo, sino que además interrogarse en cómo y por qué lo está haciendo; por esto, al hacerse partícipe del fenómeno en estudio debe reflexionar en torno a todas a estas preguntas. En este sentido, el proceso de reflexividad del investigador para comprender el entorno social en que se desenvuelve su investigación es medular en la $\mathrm{TFC}^{26}$. Por lo expuesto, es prácticamente imposible ser neutral ante la pobreza de las personas mayores que trabajaron gran parte de sus vidas en labores informales, cómo esta pobreza influye en sus actuales estados de salud, en qué medida la falta de oportunidades académicas condicionó su actual realidad, entre muchas otras interrogantes que irán nutriendo sus respuestas a partir de memos, notas de campo y por supuesto, la entrevista intensiva. 
Desde la TFC se reconocen las problemáticas que el investigador debe tener presente, ya que se considera que las investigaciones deben tener como propósito alcanzar la justicia social que necesitan las comunidades, pues a diferencia de la teoría crítica que tiene como objetivo avanzar en la justicia social, la TFC brinda un método para llevar a cabo investigaciones que aborden injusticias sociales ${ }^{43}$. Precisamente, Charmaz ahonda en estas problemáticas en su trabajo publicado el 2014; "Miradas desde los marginados: voces, silencios y sufrimiento" ${ }^{44}$, en el que establece que la marginación puede abarcarse en la investigación cualitativa a partir de las enfermedades crónicas, ya que reflejan en estas historias barreras y exclusiones de las personas que las padecen. Su reflexión se enfoca en que las personas marginadas por su condición de salud incurren en sufrimiento, por ello, la TFC debe tener como propósito a partir de sus investigadores, aumentar el poder transformador de la investigación cualitativa con esfuerzos colectivos de investigadores con intereses comunes o, lo que también es válido, hacerlo a partir de investigaciones innovadoras, incisivas y exhaustivas que rompan las barreras de la exclusión.

El rol de los investigadores que utilicen la TFC deben comprender a los participantes de sus investigaciones en el contexto de sus vidas; incluidas sus acciones, y sus preocupaciones como actores políticos y públicos ${ }^{45}$. Ante esto, el investigador en enfermería que desarrolla TFC, debe estar consciente que las personas pese a estar en condiciones desfavorables que les impidan tener una participación plena como otros grupos sociales, no tienen por qué renunciar a su condición de actores políticos, es por esto que el investigador se convierte en un nexo que permite dar una construcción a su realidad y a una teoría que permita dar mayor comprensión a sus contextos sociales, para que, en definitiva, se abran los canales que permitan sacar de la exclusión, marginalidad o invisibilidad a las personas mayores y sus problemas.

En base a lo anterior, se comprende la importancia de incorporar la reflexividad en investigaciones que utilicen la TFC, pues en definitiva es lo que permite al investigador tomar conciencia que en el conocimiento que construya, debe considerar los contextos sociales para brindar una interpretación de los fenómenos cómo los aprecian las personas y él mismo como investigador ${ }^{46}$.

\section{Reflexiones finales}

Es innegable el posicionamiento que ha adquirido la TF en campos como la enfermería, la sociología, la educación, la psicología y otros campos de las ciencias sociales, independiente de las diferentes interpretaciones y corrientes elaboradas desde la TF a través de las últimas décadas ${ }^{6}$. Una de las principales ventajas que presenta respecto a otras perspectivas, es la generación de teorías sobre fenómenos poco estudiados $^{47,48}$. En las ciencias de la salud, la metodología de la TF es usada de manera considerable ${ }^{49}$, principalmente por su flexibilidad y legitimidad adquirida que la hace atractiva para los investigadores cualitativos, con variados intereses teóricos y sustantivos $^{7}$. Esto ha llevado incluso a plantear que su método ha generado un amplio desarrollo en varias áreas innovadoras, de acuerdo con la enfermera Janice Morse $^{50}$, la TF permite no sólo documentar el cambio al interior de grupos sociales, sino que también comprender el proceso central para ese cambio.

Uno de los aportes destacados de la TFC es posicionar al investigador en un rol clave en la co-construcción de la teoría, como partícipe del proceso de investigación en conjunto con sus participantes como sujeto de cambio social. Lempert ${ }^{51}$ contribuye a esta reflexión al momento de plantear en el debate el posicionamiento social que presenta el investigador, que fue desestimado por los investigadores clásicos de la TF, por lo que su responsabilidad en abogar por los grupos sociales vulnerables, marginados y excluidos, como las personas mayores, se vuelve casi una obligación ética, en la que el responsable de llevar a cabo una investigación cualitativa con este enfoque, debe tener muy presente en sus decisiones de acuerdo a sus procesos reflexivos. Lo anterior, explicado por la enfermera Carmen De la Cuesta-Benjumea, implica que; "la reflexividad convierte al investigador en actor de su estudio e instrumento de la indagación con que construye el diseño, como producto de las decisiones que toma” ${ }^{52}$.

De esta manera, los investigadores en las ciencias de la enfermería que trabajen con temas sensibles para las personas mayores como grupo social vulnerable, deben interceder por generar una discusión al 
interior de la comunidad científica y en la sociedad, que logre dar a conocer sus necesidades y expectativas con relación a las problemáticas que los margina, excluye y condiciona su situación de salud. Hoy en día esta situación se vuelve un real desafío para los investigadores de enfermería que utilicen TFC, al exigir y denunciar a partir de sus investigaciones, la obligación que deben tener los Estados por promover y defender los derechos de las personas mayores, máxime por el proceso que se está viviendo hoy en la región, con la ratificación de la "Convención Interamericana sobre la Protección de los Derechos Humanos de las Personas Mayores", la que corresponde a un consenso actualizado de la realidad de las personas mayores en un contexto latinoamericano, ya que garantiza como principales derechos la participación, seguridad social, salud, bienestar y cuidado de las personas vulnerables ${ }^{39}$.

Del mismo modo, la TFC brinda las herramientas e instaura claramente el posicionamiento que deben adquirir de antemano los investigadores en enfermería, que la utilicen como referente ético, de la lucha y denuncia contra las injusticias sociales.

Esto sin duda, es posible, en la medida que los investigadores se involucren en indagar temas relevantes de las sociedades en las que viven. Un ejemplo de esto, está en cómo se alcanzó en Uruguay el actual Sistema Nacional de Cuidados, mediante la fusión de las estrategias de la sociedad civil con los insumos y participación de investigadores, en la elaboración y posicionamiento de un discurso social fundamentado, que logró permear a la sociedad civil, fuerzas políticas y actores gubernamentales la necesidad de la construcción de políticas de cuidados. Esto fue logrado al posicionar en la agenda pública, a los cuidados de las personas vulnerables como un problema social ${ }^{53}$.

Lo anterior demuestra que los cambios dirigidos a mejorar las condiciones de las personas mayores a gran escala son posibles, por lo que los investigadores de las ciencias de la enfermería deben abastecerse de las metodologías y herramientas, que los posicione como un actor social relevante en sus respectivos países; la TFC contribuye con su metodología, mientras que los investigadores en enfermería han de saber utilizar estratégicamente estas herramientas, en pro de visibilizar las injusticias que afectan a los grupos excluidos y que repercute en el bienestar de ellos.

\section{Responsabilidades éticas}

Protección de personas y animales. Los autores declaran no haber realizado experimentos en seres humanos ni en animales para el desarrollo de esta investigación, ya que se trata de una de tipo documental. Financiamiento. Ninguno.

Conflicto de intereses. Los autores declaran no tener conflicto de intereses.

\section{Referencias}

1. Collins R. Cuatro tradiciones sociológicas. México: Universidad Autónoma Metropolitana; 1996.

2. Denzin NK, Lincoln YS. Introduction: The discipline and practice of qualitative research. En: Denzin NK, Lincoln YS, (Eds.). The Sage handbook of qualitative research. Thousand Oaks: Sage Publications Ltd; 2005. p.1-32.

3. Castro R. Teoría social y salud. Buenos Aires: Lugar Editorial, Universidad Nacional Autónoma de Mexico; 2011. p.59-92.

4. Glaser BG, Strauss AL. The discovery of grounded theory: Strategies for qualitative research. U.S.A.: Aldine Transaction; 1967.

5. Mella-Valenzuela O. Metodología cualitativa en ciencias sociales y educación: orientaciones teóricometodológicas y técnicas de investigación. Santiago: Primus; 2003.

6. Creswell JW. Qualitative inquiry and research design: Choosing among five approaches. Thousand Oaks: SAGE publications; 2007.

7. Charmaz K. Constructing grounded theory. A practical guide through qualitative analysis. London: SAGE publications; 2006. 
8. Bonilla-García MA, López-Suárez AD. Ejemplificación del proceso metodológico de la teoría fundamentada. Cinta moebio. 2016; (57): 305-15.

http://dx.doi.org/10.4067/S0717-554X2016000300006

9. Charmaz K. Grounded theory. En: Ritzer G, (Ed.). The blackwell encyclopedia of sociology. Oxford: Blackwell Publishing Ltd; 2007. p.2023-7.

10. Moll LR, Cott CA, Nixon S. Qualitative evidence in chronic, disabling conditions (childhood-or early-onset physical impairment). En: Olson K, Young RA, Schultz IZ, (Eds.). Handbook of qualitative health research for evidence-based practice. New York: Springer-Verlag; 2016. p.411-25. https://doi.org/10.1007/978-1-4939-2920-7

11. Centeno-Silva R. Revisión de los enfoques de la teoría fundamentada y su aplicación en la investigación académica. En: Silva-Pacheco J, Graterol EA, (Comps.). Retos de la investigacion en la gerencia. Caracas: Editorial Guardagujas; 2014. p.127-54. https://doi.org/10.13140/rg.2.1.2986.6727

12. Apramian T, Cristancho S, Watling C, Lingard L. (Re)Grounding grounded theory: a close reading of theory in four schools. Qual Res. 2017; 17(4): 359-76. https://doi.org/10.1177/1468794116672914

13. Mercado-Martínez FJ. El proceso de análisis de los datos en una investigación sociocultural en salud. En: Mercado-Martínez FJ, Torres- López TM, (Eds.). Análisis cualitativo en salud. Teoría, método y práctica. México D.F.: Universidad de Guadalajara; 2000. p.47-72.

14. Taghipour A. Adopting constructivist versus objectivist grounded theory in health care research: A review of the evidence. Journal of Midwifery and Reproductive Health. 2014; 2(2): 100-4. https://doi.org/10.22038/jmrh.2014.2434

15. Hood J. Orthodoxy vs. power: The defining traits of grounded theory. En: Bryant A, Charmaz K, (Eds.). The Sage Handbook of grounded theory. London: SAGE Publications; 2007. p.151-64.

16. Levers MJD. Philosophical paradigms, grounded theory, and perspectives on emergence. Sage Open. 2013; 3(4): 1-6. https://doi.org/10.1177/2158244013517243

17. Charmaz K. Grounded theory: objectivist and constructivist methods. En: Denzin NK, Lincoln YS, (Eds.). Handbook of qualitative research. $2^{\text {nd }}$ ed. Thousand Oaks: SAGE Publications; 2000. p. 509-35.

18. Charmaz K. Constructivist grounded theory. J Posit Psychol. 2017; 12(3): 299-300. https://doi.org/10.1080/17439760.2016.1262612

19. Wiener C. Making teams work in conducting grounded theory. En: Bryant A, Charmaz K, (Eds.). The Sage Handbook of grounded theory. London: SAGE Publications; 2007. p.293-310.

20. Gibbs GR. Analyzing qualitative data. London: SAGE Publications; 2007. https://dx.doi.org/10.4135/9781849208574

21. Charmaz K.Teaching theory construction with initial grounded theory tools: A reflection on lessons and learning. Qual Health Res. 2015; 25(12): 1610-22. https://doi.org/10.1177/1049732315613982

22. Böhm A. Theoretical coding: Text analysis in grounded theory. En: Flick U, Kardorff E, Steinke I, (Eds.). A companion to qualitative research. London: SAGE Publications; 2004. p.270-5.

23. Charmaz K. Grounded theory in the 21 st century: Applications for advancing social justice studies. En: Denzin NK, Lincoln YS, (Eds.). The Sage Handbook of qualitative research. Thousand Oaks: Sage Publications; 2005. p.507-36.

24. Flick U. Managing quality in qualitative research. London: SAGE Publications; 2007.

25. Dowling M. Reflexivity. En: Given LM, (Ed.). The SAGE Encyclopedia of qualitative research methods. Thousand Oaks: SAGE Publications; 2008. p.747-8.

26. Charmaz K. Constructionism and the grounded theory method. En: Holstein JA, Gubrium JF, (Eds.). Handbook of constructionist research. New York: The Guilford Press; 2008. p.397-412.

27. HallWA, Callery P. Enhancing the rigor of grounded theory: Incorporating reflexivity and relationality. Qual Health Res. 2001; 11(2): 257-72. https://doi.org/10.1177/104973201129119082 
28. Malterud K. Qualitative research: Standards, challenges, and guidelines. Lancet. 2001; 358(9280): 483-8. https://doi.org/10.1016/S0140-6736(01)05627-6

29. Busso G. Vulnerabilidad social: nociones e implicancias de política para Latinoamérica a inicios del siglo XXI. Santiago de Chile: ONU/CEPAL/CELADE; 2001.

30. United Nations. World population prospects: The 2015 revision, key findings and advance tables. New York: UN/DESA; 2015.

31. Huenchuan S, Guzmán JM. Seguridad económica y pobreza en la vejez: tensiones, expresiones y desafíos para el diseño de políticas. Notas de Población. No.83. Santiago de Chile: CEPAL/CELADE; 2007.

32. Organización Mundial de la Salud. Informe mundial sobre el envejecimiento y la salud. Ginebra: OMS; 2015.

33. The Organisation for Economic Co-operation and Development. Health at a glance 2015: OECD indicators. Paris: OECD Publishing; 2015. http://dx.doi.org/10.1787/health_glance-2015-en

34. Del Popolo F. Características sociodemográficas y socioeconómicas de las personas de edad en América Latina. Santiago de Chile: CELADE-FNUAP/CELADE; 2001.

35. Martínez R, Trucco D, Palma A. El analfabetismo funcional en América Latina y el Caribe. Panorama y principales desafíos de política. Santiago de Chile: CEPAL; 2014.

36. World Health Organization. A conceptual framework for action on the social determinants of health. Social determinants of health discussion paper 2. Debates, policy and practice, case studies. Geneva: WHO; 2010.

37. Organización Mundial de la Salud. Cerrando la brecha: la política de acción sobre los determinantes sociales de la salud. Conferencia mundial sobre los Determinantes Sociales de la Salud. Documento de trabajo. Ginebra: OMS; 2011.

38. Gobierno de Chile/Ministerio de Desarrollo Social/Observatorio Social. Panorama Casen. Dependencia funcional en las personas mayores. Santiago de Chile: Gob. cl; 2016.

39. Organización de Estados Americanos. Convención Interamericana sobre la protección de los derechos humanos de las personas mayores. Washington, D.C: OEA; 2015.

40. Sonnicksen J. Dementia and representative democracy: Exploring challenges and implications for democratic citizenship. Dementia. 2016; 15(3): 330-42.

https://doi.org/10.1177/1471301216638998

41. Radermacher H, Sonn C, Keys C, Duckett P. Disability and participation: It's about us but still without us! J Community Appl Soc Psychol. 2010; 20(5): 333-46. https://doi.org/10.1002/casp.1039

42. Pautassi LC. El cuidado como cuestión social desde un enfoque de derechos. Santiago de Chile: NN.UU.; 2007.

43. Charmaz K. The power of constructivist grounded theory for critical inquiry. Qual Inq. 2016; 23(1): 34-45. https://doi.org/10.1177/1077800416657105

44. Charmaz K. Views from the margins: Voices, silences, and suffering. Qual Res Psychol. 2008; 16(1): 7-18. https://doi.org/10.1080/14780880701863518

45. Charmaz K. Special invited paper: Continuities, contradictions, and critical inquiry in grounded theory. Int J Qual Methods. 2017;16(1):1-8. https://doi.org/10.1177/1609406917719350

46. Hesse-Biber SN. Teaching grounded theory. En: Bryant A, Charmaz K, editors. The Sage Handbook of grounded theory. London: SAGE Publications;2007. p.311-38.

https://dx.doi.org/10.4135/9781848607941.n15

47. De la Cuesta-Benjumea C. La teoría fundamentada como herramienta de análisis. Cult. cuid. 2006; 20(2): 136-40. https://doi.org/10.14198/cuid.2006.20.19

48. Lúquez-de Camacho P, Fernández-de Celayarán O. La teoría fundamentada: precisiones epistemológicas, teóricoconceptuales, metodológicas y aportes a las ciencias. Cumbres. 2016; 2(1): 101-14. 
49. Ulin PR, Robinson ET, Tolley EE. Investigación aplicada en salud pública. Métodos cualitativos. Washington, D.C.: OPS; 2006.

50. Morse JM. Tussles, tensions, and resolutions. En: Morse JM, Stern PN, Corbin J, Bowers B, Charmaz K, Clarke A, et al. Developing grounded theory: The second generation. Pensilvania: Routledge; 2009. p.13-22.

51. Lempert LB. Asking questions of the data: Memo writing in the grounded theory tradition. En: Bryant A, Charmaz K, (Eds.). The Sage Handbook of grounded theory. London: SAGE Publications; 2007. p.245-64.

52. De la Cuesta-Benjumea C. El investigador como instrumento flexible de la indagación. Int J Qual Methods. 2003; 2(4): 25-38.

53. Aguirre R, Ferrari F. La construcción del sistema de cuidados en el Uruguay. En busca de consensos para una protección social más igualitaria. Santiago de Chile: NN.UU.; 2014. 